\title{
Technical Steps to improve Energy Efficiency of Distribution Transformers
}

\author{
Alaa Eldin ABD EL AZIZ \\ MEEDC-Egypt \\ Meedco78@yahoo.com
}

\author{
Ahmed FATEHY \\ MEEDC-Egypt \\ Meedco78@yahoo.com
}

\author{
Nelly AHMED \\ MEEDC-Egypt \\ Nelly_7875@yahoo.com
}

\begin{abstract} efficiency improvement and presented.

\section{Introduction} methods :-

1. Substitute larger conductors for those currently in use.

2. Increase the system voltage .

3. Improve system power factor by adding shunt capacitors.

4. Add lines or feeders.

5. Add or balance phases.

6. Use energy-efficient transformers.

7. Reconfigure the electrical system.
\end{abstract}

The requirements for managing distribution systems continue to present new challenges. Electricity is one of the most vital infrastructure inputs for economic development of a country. The demand for electricity in Egypt is enormous and is growing steadily. This growth has been slower than country's economic growth. To balance this demand and supply of electricity, it is the time for electric utilities to go for energy efficient electrical equipment for huge savings as this would be utilized for future needs .Therefore, great effort has been directed to increasing the efficiency of distribution transformers and consequently reducing the losses and, hence, the operational costs. International standards, published by means of norms, define the power efficiency of transformers, taking into account the no-load losses, the load losses and capitalization equations. An increase in distributor efficiency is obtained through prior knowledge of loads per unit loading and power factor adequateness. The objective at Middle Egypt Electricity Distribution Company (MEEDC) is to offer the best service to its customers through knowledge of their system and also through the acquisition of efficient equipment. The measurements of about forty different rating transformers are analyzed and their efficiency are calculated according to energy efficiency methodology These values are compared with the ideal transformers efficient tables at the same rating. Some steps are applied to energy

Utilities improve the efficiency of their transmission and distribution system by reducing losses. Industrial and commercial users of electricity also improve the efficiency of their electrical distribution systems by reducing losses . They reduce transmission and distribution losses by using the following seven basic

While some of those modifications can be done more easily than others, the use of energy-efficient transformers is always easily accomplished. But if utilities' distribution transformers are not high-efficiency , I think that is the time to improve their efficiency .

\section{Transformer Locations}

There are basically three locations and types of transformers : generation, transmission, and distribution .Fig 1 illustrates the connection between the generation and distribution systems 


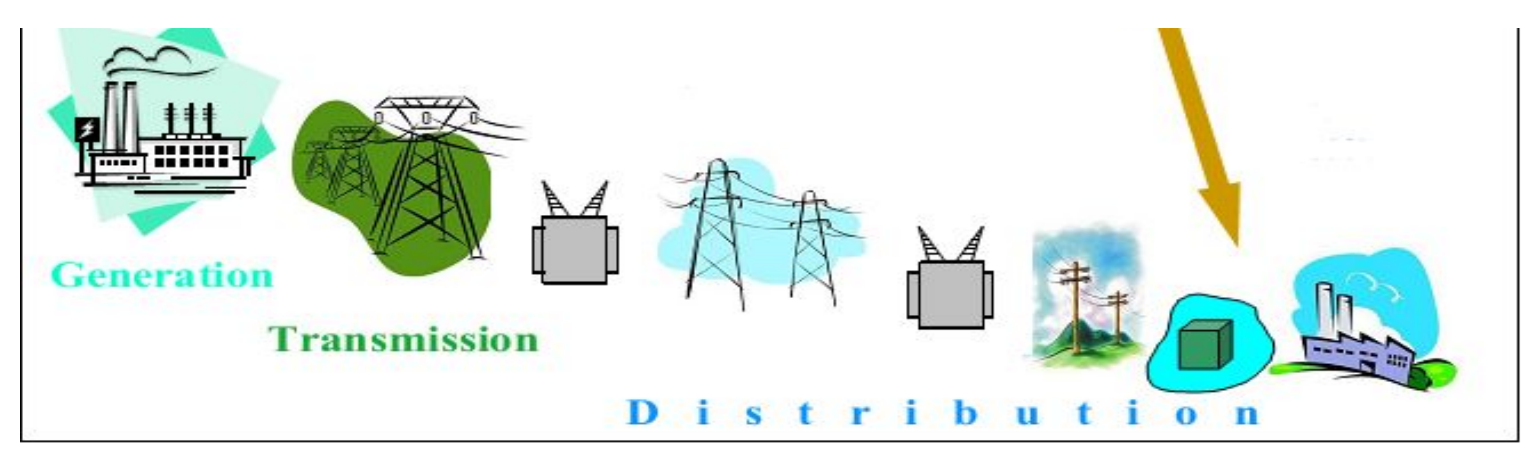

In a distribution system, distribution transformers, because of their great numbers, contribute significantly more losses than substation transformers. Basically, the issue comes down to weighing the value of efficiency improvements against the increased capital cost of energy - efficient transformers. Using energy - efficient transformers has the benefit of reducing energy consumption and thus reducing the need to operate generators that dump heat and carbon dioxide into the atmosphere. Low- efficiency transformers affect the environment by requiring increased generation to supply the increased transformer losses. This wasted generation causes increased carbon dioxide in the air and contributes to so -called greenhouse effect.

\section{What Is Transformer Efficiency?}

The efficiency of any electrical machine is defined as the ratio of power input to power output, So the efficiency can be defined by the following formula :

$\%$ Efficiency $=$ Output KVA $\times 100$

Input KVA

$\%$ Efficiency $=$ Output KVA $\times 100$

Output KVA + Losses

$=\quad P \times K V A$ rating $\times \mathbf{p . f} \times \mathbf{1 0 0 0} \times 100$

$\times$ KVA rating $\times p . f \times 1000+N . L+L . L \times P 2 \times T$

Where ,

$\mathbf{P}=$ per unit loading

N.L = No Load losses in Watts.

L.L=Load losses in Watts at full load, at $75^{\circ} \mathrm{c}$

$\mathbf{T}=$ Temperature correction factor.

p.f $=$ load power factor. 
As can be seen from this formula ,transformer efficiency is improved by simply reducing the transformer losses. Substation and distribution transformers are very efficient devices .Their efficiency varies between 98 and over 99 percent.

\section{Losses in Transformers}

- No Load (Core ) losses

Whenever an AC magnetic field is generated in the steel core, it will cause an energy loss in the core material. This is " No- Load Loss " or " core Loss " of the transformer there are in turn , two components of the No Load Loss (a) hysteresis loss and (b) eddy current loss

- Load (Copper or winding) loss

The "Load loss " is generated in two windings by load current flow in the electrical resistance of the two winding, generating simple ohmic heating from the effects of current on the winding resistance . The magnetizing current in the primary is very low magnitude compared to normal load currents and will give negligible contribution to Load Loss. The Load Loss is load current dependent as can be seen in Figure 2 and scales as the square of the load current (and the load level in KVA) so that , for example, the load loss at $100 \%$ loading will be four times the load loss at $50 \%$ loading.

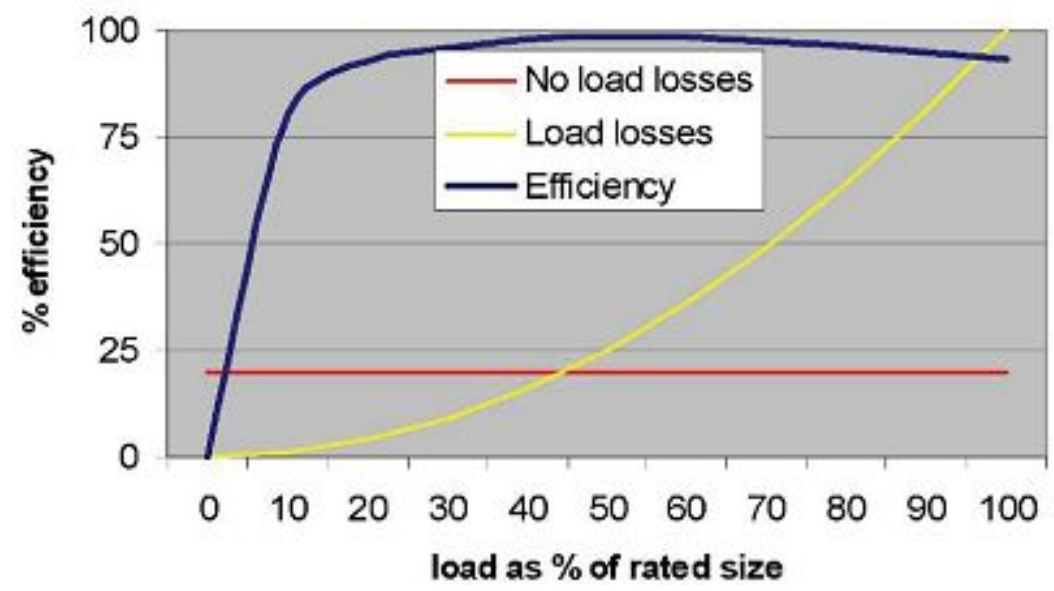

Fig 2 : Transformer loss components and power efficiency versus loading (Note that peak efficiency occurs when load loss and no- load loss are equal)

Load losses are relatively insensitive to grid voltage change. They are however very sensitive to temperature variation of the windings and this is an important consideration in analysis of the test results of efficiency determinations. Test temperature must be recorded and results normalized to specified standard temperatures in any loss and efficiency determinations. Although " copper loss " is a widely used term for Load Loss, the windings are not always made of copper. In modern distribution transformers, the secondary winding may be wound in the form of a cylindrical sheet of a aluminum This is an important consideration in adjusting losses for temperature variation. As can be seen in Fig2 the load loss becomes the dominant component when the transformer is more than about $50 \%$ loaded. The Load dependence also means that any overloading of the transformer will cause significant increase in load loss and decrease in efficiency. These types of Losses present due to the following reasons :-

- I2R losses or heat loss.

- Unoptimized location of transformers

- Lengthy single- phase lines.

- Phase imbalance.

- Loose joints 
- Low power factor loads.

- Overloading of transmission lines.

- Low Quality of insulators and conductors.

- Low quality earthing at consumer premises.

The above losses are considered "technical ", reducing them is an imperative requirement for any electric company, because if they exceed certain " allowable " levels, they can actually endanger the company's financial status.

Transformer efficiency standard in Egypt is according to global standard specifications 2009/6977

table (1) illustrate the minimum efficiency of 3 - phase (Liquid -immersed ) \& dry distribution transformers

\begin{tabular}{|c|c|c|}
\hline \multirow{2}{*}{ Rated power KVA } & \multicolumn{2}{|c|}{ Minimum efficiency } \\
\cline { 2 - 3 } & $\begin{array}{c}\text { At full load \& } \\
\text { uniformity p.f } \\
99.29\end{array}$ & $\begin{array}{c}\text { At full load \& } \\
\text { 0.8 p.f }\end{array}$ \\
\hline 25 & $98.08 \%$ & $97.58 \%$ \\
\hline 50 & $98.48 \%$ & $97.96 \%$ \\
\hline 63 & $98.32 \%$ & $97.70 \%$ \\
\hline 100 & $98.72 \%$ & $97.25 \%$ \\
\hline 160 & $98.86 \%$ & $97.43 \%$ \\
\hline 200 & $98.93 \%$ & $98.53 \%$ \\
\hline 300 & $98.99 \%$ & $98.56 \%$ \\
\hline 500 & $99.18 \%$ & $98.78 \%$ \\
\hline 100 & $99.27 \%$ & $98.92 \%$ \\
\hline 1500 & $99.29 \%$ & $98.94 \%$ \\
\hline 2000 & $99.30 \%$ & $98.97 \%$ \\
\hline 2500 & $99.34 \%$ & $99.08 \%$ \\
\hline & $99.32 \%$ & $99.03 \%$ \\
\hline
\end{tabular}

\section{Case Study}

MEEDC has about $16831 \mathrm{~km}$ over head transmission lines and $5623 \mathrm{~km}$ cables under medium tension The measurements was carried out on one redial feeder under medium voltage which supplies about forty MV/LV distribution transformers Fig 3 illustrates the power flow of this feeder.

- The measured parameters for each transformer are V,I, P.F

- The Calculated values are transformer efficiency, per unit loading, total annual energy losses KWh.

- No-Load losses and Load Losses are given from manuals according to transformer type.

The data of about forty transformers includes the rated power KVA, PF and per unit loading of each transformer are obtained as shown in graph (1). 


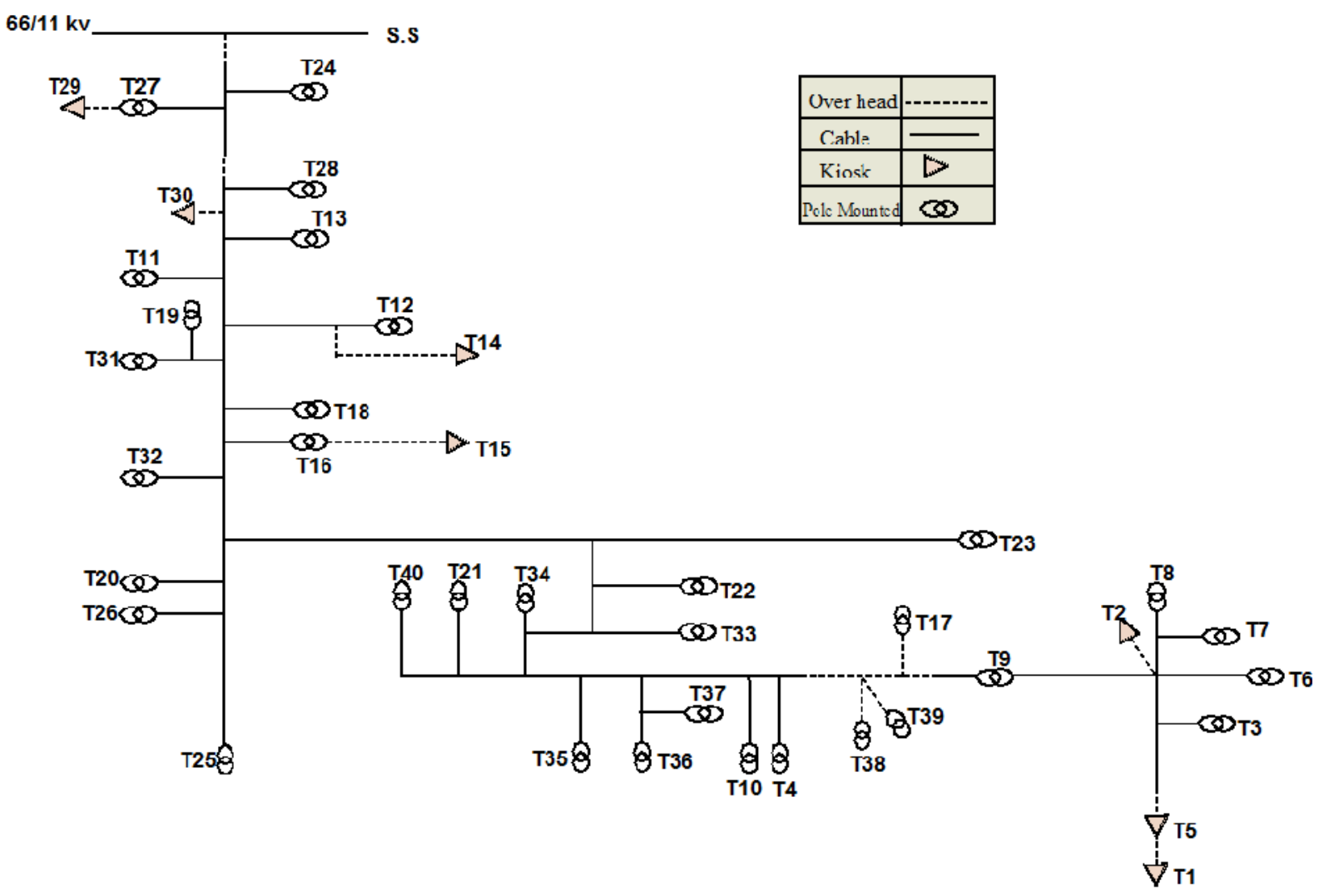

Fig 3 : Power Flow of Outgoing Redial Feeder

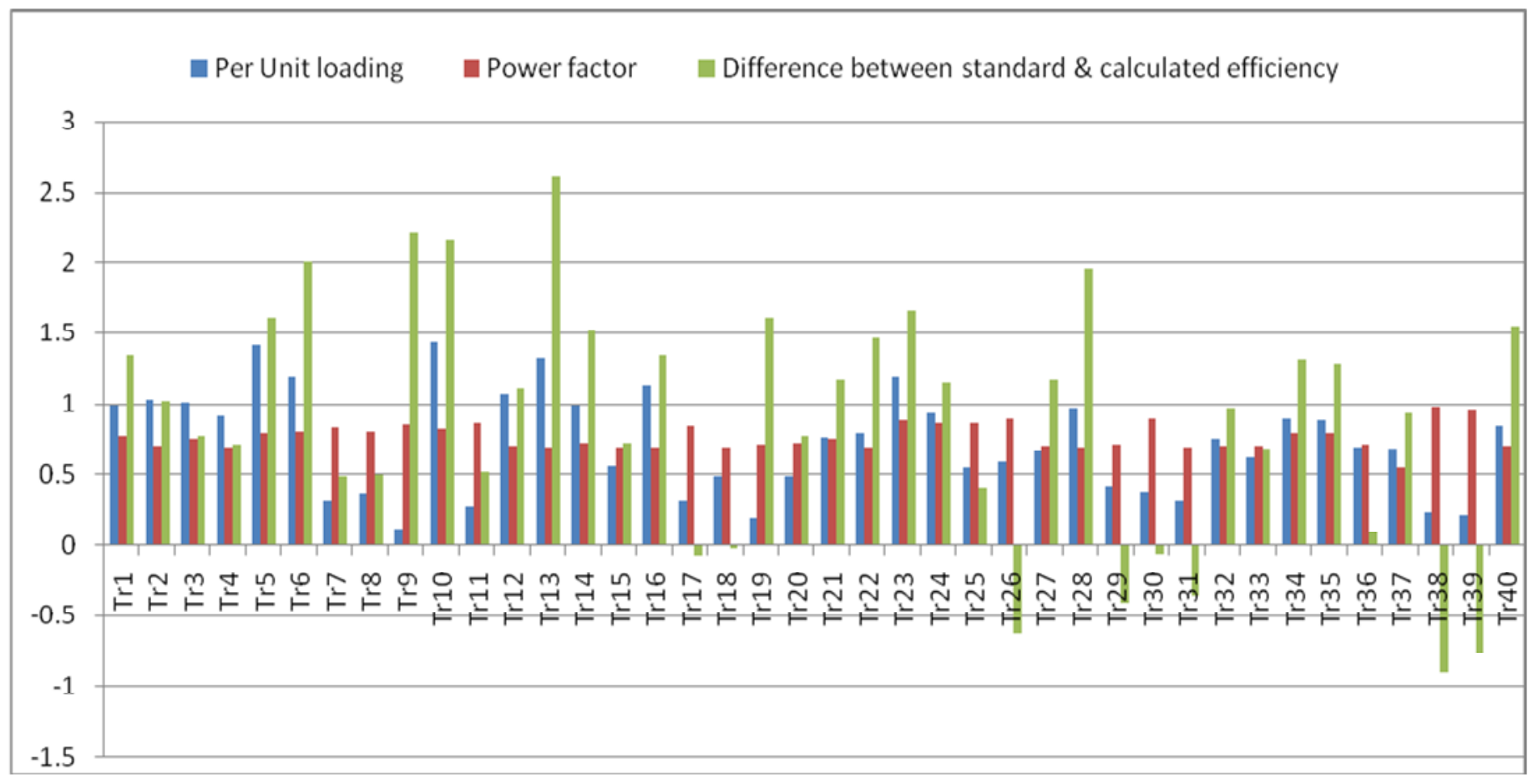

Graph (1) 
Trough the measurements of these forty distribution transformers we can calculate the efficiency according to the transformer efficiency formula, The knowledge if this transformer is high efficiency or not was obtained by comparing the value of calculated transformers efficiency and the other value of minimum efficiency according to the global standard specifications 2009/6977 the specialist in MEEDC analyzed these data at table (1), they found :-

1- The negative sign of the difference value at eight transformers indicated their calculated efficiency is higher than the minimum value at the standard. This is due to some factors such as the per unit loading of these transformers are in the acceptable range of loading transformers according to the relation between load\% of rated size and \% efficiency which illustrated at Fig2, the second factor for increasing the efficiency is the high value of power factor (ex.Tr38,T39).

2- As long as the high per unit loading the low efficiency percentage like $(\operatorname{Tr} 1, \operatorname{Tr} 2, \operatorname{Tr} 5 \ldots)$ in this case study Note that the difference value between standard efficiency and calculated value is higher than $>1$, Besides the high loading transformer percentage represents more dangerous related to the transformer, so we had to replace these transformers.

3- No-load losses and load losses were calculated for each transformer and by accumulating values along this feeder . Total Annual Energy Losses (TAEL) is about $220754 \mathrm{KWh}$, the cost of this losses is about 146719 LE .The calculation were done according to the load factor equals to 0.3 .Some steps should be applied to improve the transformer efficiency and decrease the losses.

Step1 nine of high loading transformers were replaced by others of larger rated power (K.V.A).This leads to increase transformer efficiency and decrease load losses as follows :-

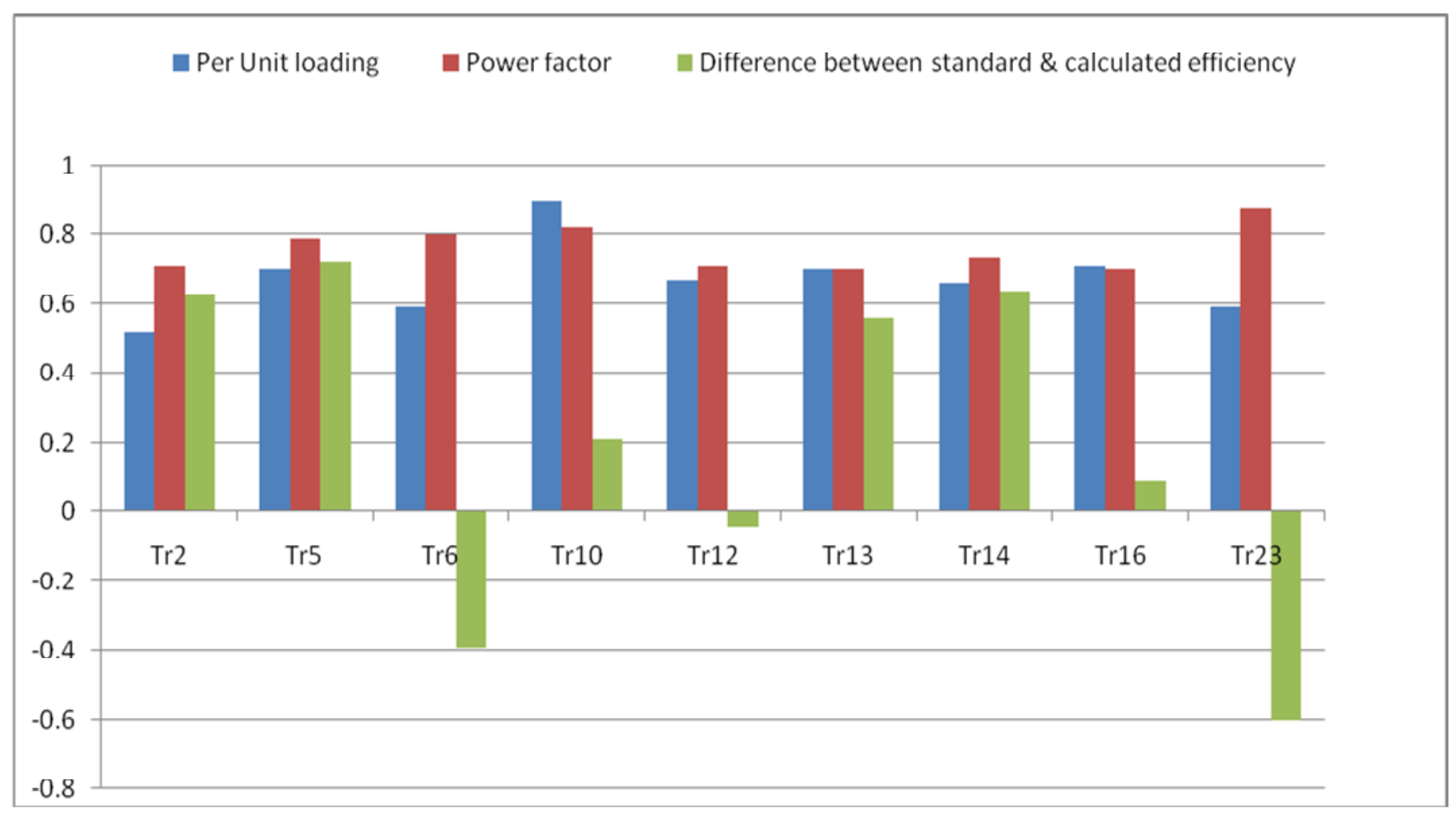


Total Annual Energy losses (TAEL) became about $198793 \mathrm{KWh}$, the cost of this losses was about 132005 LE.TAEL was decreased by $10 \%$ through applying this procedure .In addition the high loading transformers must be replaced to avoid happening any risk may be done at transformers.

$\underline{\text { Step2 }}$ unbalanced three phase current was rearranged and the measurements were updated for 6 transformers as follows :-

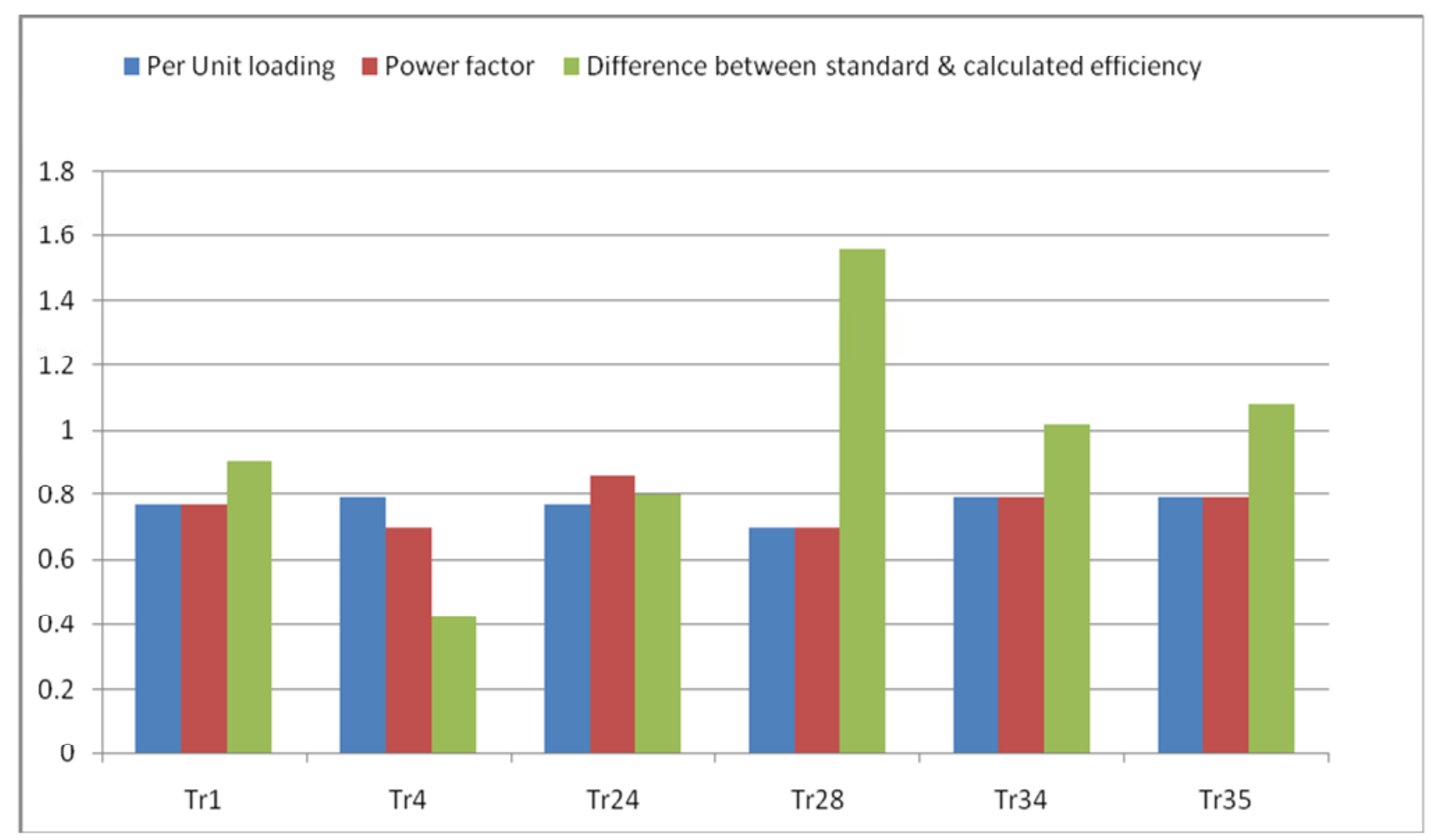

The current balance was done after rearrangement the distribution of three phase current ,So the value of per unit loading was decreased, Hence the transformer efficiency increased, But there was some transformers such as (T28,T34,T35) need more procedures to increase their efficiency .Total annual energy losses (TAEL) became about $187739 \mathrm{kwh}$, the cost of this losses was about $124799 \mathrm{LE}$. TAEL was decreased by 15\% through applying this procedure .

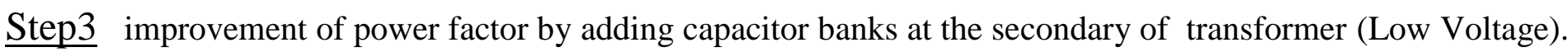
But because of the large number of the existing transformers adding capacitors banks at low voltage is not economic and very expensive .So we added shunt capacitor $300 \mathrm{KVAR}$ at medium tension Hence the power factor was improved at the outgoing feeder.

\section{Conclusion}

High - efficiency transformers are available commercially today .The transformer manufacturers are able to provide myriad choices of transformers of varying losses .It all depends on how high the transformer purchaser values losses. As utilities and other large purchasers have insisted on lower - loss high- efficiency transformers, the transformer manufacturers have been able to develop transformers with lower losses .The reduction in losses over the years have come by improving the material and construction of the core and coils of distribution transformers. By using low-loss amorphous steel core transformers and widespread use of evaluation methodologies, a reduction in no-load and load loss was occurred in the transformer. Hence we 
can buy high- efficiency transformers or make some technical procedures to improve the transformers efficiency which are already exist in the distribution network such as :-

- Regular measurements of transformers.

- Revise efficiency calculation criteria.

- Revise transformer temperature.

- Avoid over - sizing of Transformers

\section{References}

[1] Reduction of Energy Losses in Electrical Distribution System 24st International Conference on Electricity Distribution (CIRED 2013) by Hamid EMARA KASSEM , Mohammed BADR , Salwa ALI AHMED

[2] Best Practice Manual of Transformers prepared for Bureau of energy efficiency, Indian Renewable Energy Development Agency by Devki energy consultancy Pvt.Ltd.,

[3] Optimizing Energy Efficiency Standards For Low Voltage Distribution Transformers by Kenneth Duane Harden , May 2011 Purdue University Fort Wayne,Indiana.

[4] Energy Efficient Transformers book by BARRY W . Kennedy. 